\section{Equivalent Dose and Risk of Exposure Induced Cancer Death of Different Organs due to Various Image Techniques of EOS Imaging System}

\author{
Nima Hamzian ${ }^{\oplus}$, Saeid Afereydoon², Mahdi Ghorbani3*e, Seyed \\ Mohammad Jalil Abrisham4, Zahra Roozmand5, Sepideh Abdollahi- \\ Dehkordi', Morteza Sepehr Javan7, Mohammad Reza Deevband³
}

\begin{abstract}
Background: Euronext Paris Advanced Orthopedic Solutions (EOS) system is a new radiography system, capable of obtaining two-dimensional and three-dimensional images from bony structures in the body.

Objective: The aim of this study is to estimate equivalent dose and the risk of exposure induced cancer death (REID) in different organs of body due to EOS imaging system.

Material and Methods: In this experimental study, totally 120 patients were evaluated for various imaging techniques of lower limb, full spine and whole body. Equivalent dose and REID for colon, liver, lung, stomach, breast, bladder, ovary, blood cells (leukemia) and other organs were calculated using PCXMC software (version 2.0.1.2) based on Monte Carlo simulation of X-ray and human phantoms. The data on imaging technique, including age, sex, $\mathrm{kVp}$, dose area product (DAP), $\mathrm{mA}$, focal to detector distance were introduced as the input of PCXMC.
\end{abstract}

Results: The maximum equivalent dose (mSv) due to EOS imaging system, was estimated for the bladder $0.240 \pm 0.066$ for the full body technique and $0.240 \pm 0.093$ for the lower limb technique, respectively, in both males and females. The maximum organ REID (incidence per million) due to EOS imaging system was estimated for lungs as $2.59 \pm 1.0$ and $2.53 \pm 0.9$, for the full body technique in both males and females, respectively.

Conclusion: Generally, the equivalent dose and REID by EOS imaging system in different organs of body is low due to the low radiation dose received by the body in different techniques and views.

Citation: Hamzian N, Afereydoon S, Ghorbani M, Abrisham SMJ, Roozmand Z, Abdollahi-Dehkordi S, Sepehr Javan M, Deevband MR. Equivalent Dose and Risk of Exposure Induced Cancer Death of Different Organs due to Various Image Techniques of EOS Imaging System. J Biomed Phys Eng. 2021;11(3): 289-296. doi: 10.31661/jbpe.v0i0.2012-1242.

\section{Keywords}

Radiation Dosage; Radiography; Radiation Effects; Risk of Exposure induced Cancer Death; EOS Imaging System

\section{Introduction}

$\mathrm{I}$ nvestigation of deformity in spinal and bone structures via radiological techniques is a vital tool in the diagnosis of pathological changes in body and surgeons use this tool to select the best treatment with least invasive procedures. In conventional radiography with X-ray, computed tomography and digital radiography are standard modalities for
${ }^{1} \mathrm{PhD}$, Department of

Medical Physics, School of

Medicine, Shahid Sadoughi

University of Medical Sci-

ences, Yazd, Iran

${ }^{2} \mathrm{MSc}$, Department of

Medical Physics, School of

Medicine, Shahid Sadoughi

University of Medical Sci-

ences, Yazd, Iran

${ }^{3} \mathrm{PhD}$, Department of

Biomedical Engineering and

Medical Physics, School of

Medicine, Shahid Beheshti

University of Medical Sci-

ences, Tehran, Iran

${ }^{4} \mathrm{MD}$, Department of EOS

Imaging, Shahid Sadoughi

General Hospital, Shahid

Sadoughi University of

Medical Sciences, Yazd, Iran

${ }^{5} \mathrm{BSc}$, Department of

Medical Physics, School of

Medicine, Shahid Sadoughi

University of Medical Sci-

ences, Yazd, Iran

${ }^{6} \mathrm{MSc}$, Department of

Medical Physics, School of

Medicine, Iran University of

Medical Sciences, Tehran,

Iran

${ }^{7} \mathrm{BSC}$, Department of EOS

Imaging, Shahid Sadoughi

General Hospital, Shahid

Sadoughi University of

Medical Sciences, Yazd, Iran

*Corresponding author: Mahdi Ghorbani

Assistant Professor, Department of Biomedical

Engineering and Medica

Physics, School of Medi-

cine, Shahid Beheshti

University of Medical

Sciences, Tehran, Iran

E-mail: mhdghorbani@ gmail.com

Received: 6 December 2020 Accepted: 26 January 2021 
obtaining imaging information from skeletal deformities [1].

Euronext Paris Advanced Orthopedic Solutions (EOS) system is a new imaging system with capability of obtaining two-dimensional (2D) anteroposterior (AP) and lateral images from the whole body. These 2D images can then be used for the three-dimensional (3D) reconstruction of bony structures in the body as a stereo radiography tool [2]. This new radiography modality is a slot-scanning $\mathrm{X}$ ray, presented by EOS (EOS Imaging, Paris, France) and also adopted by radiology community, and has advantage of imaging with very low radiation dose as well. In this system, two sets of X-ray tubes are installed at right angles as a biplanar configuration. A multi-wire proportional chamber (MWPC) is used for the detection of the radiation and acquisition of the images, simultaneously, from different views. By this modality, it is possible to measure and analyze spine curvature, bone fracture, torsion, orientation and alignment of spine and lower limb in body. The evaluation of the accuracy, reliability and reproducibility of this system showed advantages compared to manual 2D and computed tomography (CT) modalities [3].

During recent years, various researchers have performed studies on the determination of the patient dose or risk due to EOS imaging [4-6]. These studies were performed by calculation or measurement of the patient dose or the cancer risk. There are also studies on the comparison of the patient dose or the cancer risk from different X-ray imaging modalities, including EOS, digital radiography (DR) and computed tomography (CT) [7-10]. However, to the best of our knowledge, there is not any study on the risk of the exposure induced cancer death (REID) determination for different body organs due to EOS imaging of the patients with different imaging techniques. The aim of this study is to estimate the equivalent dose and REID for different organs of body due to radiography of the patients by an EOS imaging system.

\section{Material and Methods}

In the present experimental study, 120 patients were evaluated and the techniques for imaging of the patients were whole body, full spine and lower limb. Equivalent dose and REID for colon, liver, lung, stomach, breast, bladder, ovary, blood cells (leukemia) and other organs were calculated using PCXMC (a PC Program for X ray Monte Carlo) software (version 2.0.1.2).

The patient data, including $\mathrm{kVp}$, dose area product (DAP), mA, focus to detector distance, field size, imaging technique, imaging view, age, height, weight and sex of patients were collected. Then the patient data were introduced as the input of this software and REID was calculated based on Monte Carlo simulation of X-ray and human phantoms.

\section{Patients and EOS system}

Patients referred to an EOS (EOS Imaging, Paris, France) imaging department were evaluated based on REID for different organs due to radiation received by this imaging system. In this system, the gas ionization detectors and thin grid ionization chambers are used for detection of X-ray. To decrease the effect of scattered radiation, the detector has a collimator. The detectors are perpendicular to each other to have the capability of three-dimensional imaging.

The total number of patients was 120 and in each technique, 40 patients were included in the study (20 male and 20 female). The ages of patients were in the range of 3-80 years.

The imaging techniques were full spine, lower limb, and full body. Images were acquired in lateral, anterior-posterior (AP) and posterior-anterior (PA) views, but the results were averaged for both views for each patient. The imaging was performed in Shahid Sadoughi Hospital (Yazd, Iran). The patient and the imaging technique information, including, age, sex, height, weight, $\mathrm{kVp}$, focal to detector 
Equivalent Dose; REID; EOS Imaging System

distance, dose area product (DAP), field size, filter type and thickness, and patient descent were noted and introduced in the PCXMC software for the calculation of the equivalent dose and REID for different organs. The organs included the colon, liver, lung, stomach, breast, bladder, ovary, blood cells (leukemia) and other organs.

Ethical guidelines by Shahid Beheshti University of Medical Sciences (ethics code: IR.SBMU.MSP.REC.1399.236) were followed in different steps of this research, including patient information collection, dose calculation and report of the result data. $\mathrm{kVp}$ and $\mathrm{mA}$ for different techniques (lower limb, full spine, full body) for male and female are tabulated in Table 1.

Organ dose calculation using PCXMC software

A PCXMC (version 2.0.1.2) software (STUK, Finland) [11] was used for the estimation of REID for different organs. The data of each imaging technique (including age, weight, height, imaging view, $\mathrm{kVp}, \mathrm{mA}$, field size, focus to detector distance, dose area product) are introduced as the input of this software. The software calculates effective dose, REID and other dose or risk quantities, based on Monte Carlo simulation of X-rays and human phantoms. The equivalent dose $(\mathrm{mSv})$ for different organs (based on International Commission on Radiological Protection (ICRP) 103 report [12]), kinetic energy released per unit mass (kerma), and REID for different organs can be calculated using this software. In Figure 1, a part of this software is illustrated as a sample.

\section{Results}

Mean equivalent dose $(\mathrm{mSv})$ for different organs for lower limb, full spine and full body techniques are presented in Tables 2, 3 and 4, respectively. The results were distinguished for the males and the females. The maximum equivalent dose $(\mathrm{mSv})$ due to EOS imaging system, was estimated in bladder $0.240 \pm 0.066$ for the full body technique and $0.240 \pm 0.093$ in the lower limb technique, respectively, for both the males and the females.

Mean REID for different organs for lower limb, full spine and full body techniques are presented in Table 5. The results were distinguished for the male and female patients. Due to EOS imaging radiography, the maximum organ REID (incidence per million) estimated for lungs as $2.59 \pm 1.0$ and $2.53 \pm 0.9$ in the full body technique for both males and females, respectively.

\section{Discussion}

In the present study, the equivalent dose and REID were estimated for different organs of body due to imaging of the patients using an EOS imaging system. There was difference in the equivalent dose and REID for different organs in EOS imaging. Based on the results in Table 2, the maximum equivalent dose values are obtained for bladder $(0.258$ $\mathrm{mSv})$ and prostate $(0.231 \mathrm{mSv})$ in the lower limb technique. The minimum values are for thyroid $(0.017 \mathrm{mSv})$, active bone marrow and lung $(0.063 \mathrm{mSv}$ for both) for this tech-

Table 1: Exposure parameters and average age of patient undergone different techniques of Euronext Paris Advanced Orthopedic Solutions (EOS) (lower limb, full spine, full body) for male and female.

\begin{tabular}{ccccccc} 
Gender & \multicolumn{3}{c}{ Male } & \multicolumn{3}{c}{ Female } \\
\hline Technique & Lower limb & Full spine & Full body & Lower limb & Full spine & Full body \\
\hline kVp & 80 & 93 & 110 & 85 & 95 & 106 \\
\hline mA & 205 & 243 & 250 & 220 & 245 & 243 \\
\hline Age average & 45 & 41 & 34 & 39 & 38 & 46
\end{tabular}


(a)

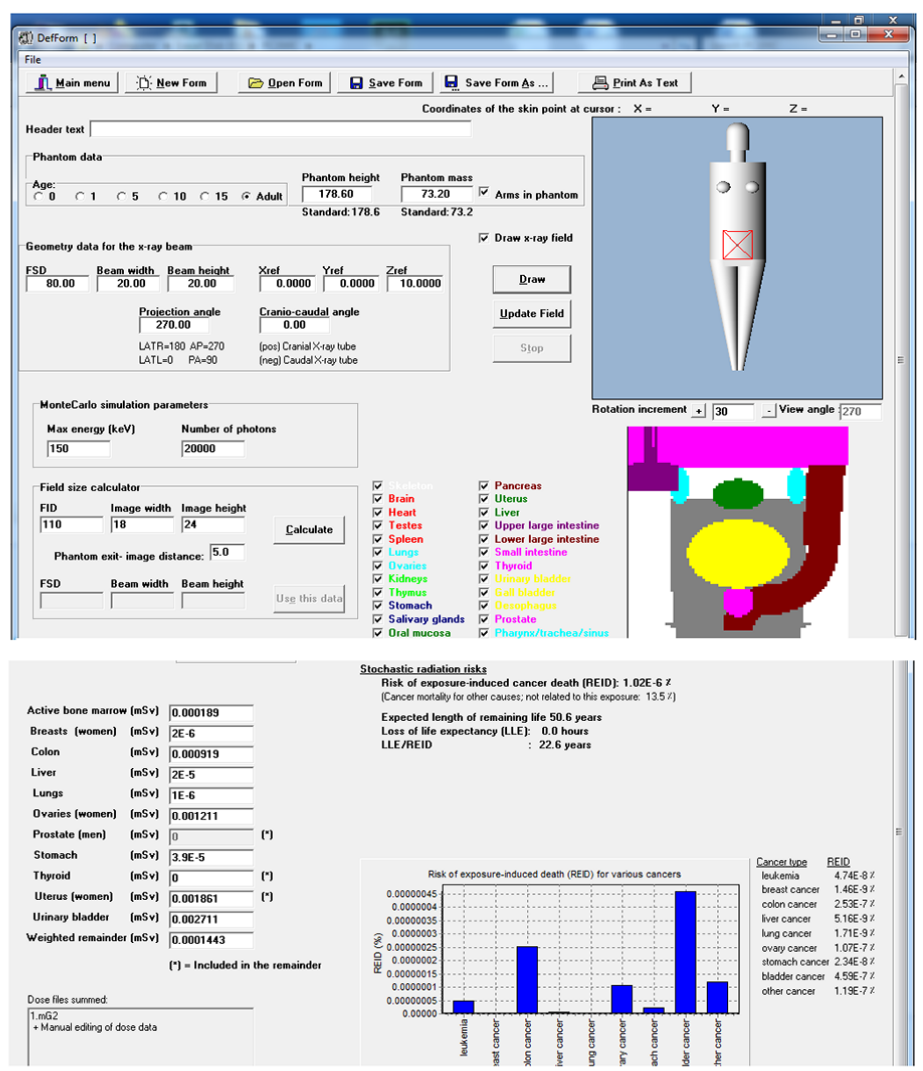

Figure 1: A part of PCXMC software. (a) For introduction of input parameters. (b) Calculated organ equivalent doses ( $\mathrm{mSv}$ ) and the risk of the exposure induced cancer death (REID).

Table 2: Equivalent dose (mSv) for different organs due to Euronext Paris Advanced Orthopedic Solutions (EOS) imaging in lower limb technique.

\begin{tabular}{cccc} 
Organ & Male & Female & Mean \\
\hline $\begin{array}{c}\text { Active bone } \\
\text { marrow }\end{array}$ & $0.060 \pm 0.021$ & $0.066 \pm 0.033$ & 0.063 \\
\hline Breast & - & $0.129 \pm 0.118$ & 0.129 \\
\hline Colon & $0.188 \pm 0.052$ & $0.203 \pm 0.080$ & 0.196 \\
\hline Liver & $0.117 \pm 0.043$ & $0.130 \pm 0.059$ & 0.123 \\
\hline Lungs & $0.057 \pm 0.053$ & $0.069 \pm 0.063$ & 0.063 \\
\hline Ovaries & - & $0.156 \pm 0.086$ & 0.156 \\
\hline Prostate & $0.231 \pm 0.063$ & - & 0.231 \\
\hline Stomach & $0.156 \pm 0.060$ & $0.158 \pm 0.091$ & 0.157 \\
\hline Thyroid & $0.015 \pm 0.004$ & $0.020 \pm 0.003$ & 0.017 \\
\hline Uterus & - & $0.184 \pm 0.074$ & 0.184 \\
\hline Bladder & $0.236 \pm 0.049$ & $0.240 \pm 0.093$ & 0.238 \\
\hline Total & 1.06 & 1.355 & 1.576
\end{tabular}

Table 3: Mean equivalent dose (mSv) for different organs due to Euronext Paris Advanced Orthopedic Solutions (EOS) imaging system in the full spine technique.

\begin{tabular}{cccc} 
Organ & Male & Female & Mean \\
\hline $\begin{array}{c}\text { Active bone } \\
\text { marrow }\end{array}$ & $0.071 \pm 0.022$ & $0.074 \pm 0.009$ & 0.072 \\
\hline Breast & - & $0.175 \pm 0.080$ & 0.175 \\
\hline Colon & $0.139 \pm 0.032$ & $0.140 \pm 0.046$ & 0.139 \\
\hline Liver & $0.169 \pm 0.087$ & $0.170 \pm 0.099$ & 0.169 \\
\hline Lungs & $0.148 \pm 0.044$ & $0.149 \pm 0.039$ & 0.148 \\
\hline Ovaries & - & $0.137 \pm 0.048$ & 0.137 \\
\hline Prostate & $0.132 \pm 0.064$ & - & 0.132 \\
\hline Stomach & $0.169 \pm 0.061$ & $0.177 \pm 0.059$ & 0.173 \\
\hline Thyroid & $0.149 \pm 0.103$ & $0.152 \pm 0.078$ & 0.150 \\
\hline Uterus & - & $0.165 \pm 0.076$ & 0.165 \\
\hline Bladder & $0.158 \pm 0.054$ & $0.167 \pm 0.071$ & 0.162 \\
\hline Total & 1.135 & 1.506 & 1.576
\end{tabular}


Equivalent Dose; REID; EOS Imaging System

nique. For the full spine technique, the maximum equivalent dose values are obtained for stomach $(0.173 \mathrm{mSv})$ and breast $(0.175 \mathrm{mSv})$ and the minimum values are related to active bone marrow $(0.072 \mathrm{mSv})$ and prostate $(0.132$ $\mathrm{mSv}$ ) (Table 3). According to Table 4, in the

Table 4: Mean equivalent dose (mSv) for different organs due to Euronext Paris Advanced Orthopedic Solutions (EOS) imaging system in the full body technique.

\begin{tabular}{cccc} 
Organ & Male & Female & Mean \\
\hline $\begin{array}{c}\text { Active bone } \\
\text { marrow }\end{array}$ & $0.077 \pm 0.026$ & $0.074 \pm 0.028$ & 0.075 \\
\hline Breast & - & $0.181 \pm 0.071$ & 0.181 \\
\hline Colon & $0.167 \pm 0.052$ & $0.163 \pm 0.058$ & 0.165 \\
\hline Liver & $0.180 \pm 0.083$ & $0.175 \pm 0.085$ & 0.177 \\
\hline Lungs & $0.136 \pm 0.047$ & $0.125 \pm 0.052$ & 0.130 \\
\hline Ovaries & - & $0.139 \pm 0.059$ & 0.139 \\
\hline Prostate & $0.135 \pm 0.052$ & - & 0.135 \\
\hline Stomach & $0.198 \pm 0.117$ & $0.173 \pm 0.086$ & 0.185 \\
\hline Thyroid & $0.160 \pm 0.072$ & $0.158 \pm 0.043$ & 0.159 \\
\hline Uterus & - & $0.172 \pm 0.056$ & 0.172 \\
\hline Bladder & $0.240 \pm 0.066$ & $0.234 \pm 0.038$ & 0.237 \\
\hline Total & 1.293 & 1.594 & 1.775
\end{tabular}

full body technique, the maximum values are related to bladder, stomach and breast $(0.237$, 0.185 and $0.181 \mathrm{mSv}$, respectively).

Based on the data in Table 5, the maximum and minimum REID (estimated death in the population per million) are related to colon $(1.46 \%)$ and ovaries $(0.13 \%)$ in the lower limb technique. Moreover, for the full spine technique, the maximum and minimum values were obtained for lungs $(2.35 \%)$ and ovaries $(0.1 \%)$, respectively. For the full body technique, the maximum REID (estimated deaths in population per million) is estimated for lung $(2.56 \%)$ and the minimum is related to ovaries $(0.34 \%)$ (Table 5$)$. The comparison of equivalent dose and REID values shows that those organs with the maximum or minimum equivalent dose are not the same as those organs with the maximum or minimum REID. This can be due to the fact that there are some limitations in calculation of REID by the PCX$\mathrm{MC}$ software. In other words, this software presents REID only for some specific organs. Additionally, the risk coefficients vary for different organs and therefor while an organ has the maximum equivalent dose, it may not have the maximum REID. For example, for the full body technique, the maximum equivalent dose

Table 5: Mean risk of exposure induced cancer death (REID) $\left(\times 10^{-6}\right)$ for the different organs due to Euronext Paris Advanced Orthopedic Solutions (EOS) imaging system in lower limb, full spine and full body techniques. REID was presented herein in terms of number of deaths in the population per million.

Lower limb

Full spine

Full body

\begin{tabular}{cccccccccc}
\hline Organ & Male & Female & Total & Male & Female & Total & Male & Female & Total \\
\hline Breast & - & $0.68 \pm 0.20$ & 0.68 & - & $1.30 \pm 1.30$ & 1.3 & - & $1.39 \pm 0.6$ & 1.39 \\
\hline Colon & $1.38 \pm 0.25$ & $1.54 \pm 1.50$ & 1.46 & $0.56 \pm 0.20$ & $0.60 \pm 0.20$ & 0.58 & $1.42 \pm 0.7$ & $1.39 \pm 0.3$ & 1.40 \\
\hline Liver & $0.49 \pm 0.30$ & $0.60 \pm 0.05$ & 0.54 & $1.08 \pm 0.40$ & $1.09 \pm 0.50$ & 1.08 & $1.19 \pm 0.06$ & $1.14 \pm 0.2$ & 1.16 \\
\hline Lungs & $0.65 \pm 0.07$ & $0.82 \pm 0.40$ & 0.73 & $2.33 \pm 1.40$ & $2.37 \pm 0.20$ & 2.35 & $2.59 \pm 1.0$ & $2.53 \pm 0.9$ & 2.56 \\
\hline Ovaries & - & $0.13 \pm 0.10$ & 0.13 & - & $0.10 \pm 0.10$ & 0.1 & - & $0.34 \pm 0.1$ & 0.34 \\
\hline Stomach & $0.98 \pm 0.72$ & $1.13 \pm 0.70$ & 1.05 & $1.24 \pm 0.90$ & $1.25 \pm 0.40$ & 1.24 & $1.38 \pm 0.3$ & $1.80 \pm 0.4$ & 1.33 \\
\hline Bladder & $0.48 \pm 0.17$ & $0.56 \pm 0.20$ & 0.52 & $0.40 \pm 0.20$ & $0.30 \pm 0.10$ & 0.3 & $0.52 \pm 0.09$ & $0.46 \pm 0.2$ & 0.49 \\
\hline Leukemia & $0.22 \pm 0.14$ & $0.30 \pm 0.20$ & 0.26 & $0.20 \pm 0.09$ & $0.25 \pm 0.04$ & 0.22 & $0.39 \pm 0.09$ & $0.34 \pm 0.1$ & 0.36 \\
\hline Other & $0.96 \pm 0.66$ & $0.99 \pm 0.10$ & 0.97 & $1.47 \pm 0.30$ & $1.55 \pm 0.40$ & 1.51 & $1.59 \pm 0.60$ & $1.50 \pm 0.30$ & 1.54
\end{tabular}


values are related to bladder and stomach, but the maximum REID are related to lung and colon.

For male patients, in the lower limb imaging, the maximum and minimum equivalent dose $(\mathrm{mSv})$ values are for bladder and thyroid, respectively (Table 2). The same trend is observed for the females in this technique. This trend can be explained by considering this fact that in the lower limb technique, bladder is very close to the radiation field while thyroid is far.

For both male and female patients, in the full spine imaging technique, the maximum and minimum equivalent dose values are related to stomach and active bone marrow, respectively (Table 3). For the full body imaging technique, the maximum and minimum equivalent dose is related to bladder and active bone narrow, respectively (Table 4). For female patients, in full spine and full body technique, the breast equivalent dose was among the highest values. This can be explained by the fact that in full body technique, breast is in the radiation field. Generally, the equivalent dose in different organs of the body is low due to the radiation received by the body during EOS imaging in different imaging techniques and views.

For male patients, among these organs, in the lower limb imaging, the maximum and minimum REID values are for colon and leukemia, respectively (Table 5). For the same cases in female, the maximum and minimum REID values are related to colon and ovaries, respectively.

According to ICRP-103 commission [12], tissue weighting factor $\left(W_{T}\right)$ for tissues such as colon, lung and breast is 0.12 while this value for gonads is 0.08 ; thus, in the lower limb technique for both the males and the females, the highest REID is related to colon and it can be also explained based on the fact that colon is in the radiation field and is among the highest equivalent doses for both gender based on Table $2(0.188 \mathrm{mSv}$ for male and $0.203 \mathrm{mSv}$ for female).
As it was stated, the highest equivalent dose in lower limb technique is bladder in the males and the females $(0.236 \mathrm{mSv}$ and $0.240 \mathrm{mSv}$, respectively), but its $W_{T}$ is 0.04 , which is lower than colon thus the REID for colon is the highest.

For full spine technique, in the males, the maximum REID is related to lungs and the minimum is related to leukemia (Table 5). For the same conditions in females, the maximum and minimum are related to lungs and ovaries, respectively. For the full body technique, in males, the maximum and minimum REID values are related to lung and leukemia, respectively (Table 5). For the same conditions in females, the maximum value is related to lung and the minimum values are for ovaries and leukemia, respectively.

In the full spine and the full body techniques, lungs are located in radiation field with $W_{T}$ of 0.12 , which is the highest value among different body tissues, while $W_{T}$ of liver, which is among the highest values of equivalent dose in full spine and full body technique, is 0.04 .

Generally, the REID value by radiation in different organs of body is related to $W_{T}$, radiation field and exposure settings in different imaging techniques and views.

Different studies have shown that the patient dose in EOS radiography is lower than other radiography techniques such as CT and digital radiography [13-15]. For example, Alrehily et al. [13] reported that EOS has lower radiation exposure in scoliosis patients, in comparison with CT and normal radiography. In the study by Escott et al. [14] it was observed that radiography with EOS protocols delivers lower radiation to the patients, while it is more efficient in $\mathrm{CT}$ scan and routine radiography to assess length.

\section{Conclusion}

There was difference in equivalent dose and the risk of the exposure induced cancer death by radiation in different organs in various techniques of EOS imaging system. Generally, due 
to the low radiation dose received by the body during EOS imaging in different imaging techniques and views, the equivalent dose and the risk of the exposure induced cancer death for different organs of body are low.

\section{Acknowledgment}

The authors appreciate Shahid Beheshti University of Medical Sciences for financial support of this work (Code number: 20641) and EOS department of Shahid Sadoughi Hospital for providing the patient and imaging techniques data. The research was approved by the ethical committee of Shahid Beheshti University of Medical Sciences with a code number of IR.SBMU.MSP.REC.1399.236.

\section{Conflict of Interest}

\section{None}

\section{References}

1. Branchini M, Del Vecchio A, Gigliotti CR, Loria A, Zerbi A, Calandrino R. Organ doses and lifetime attributable risk evaluations for scoliosis examinations of adolescent patients with the EOS imaging system. Radiol Med. 2018;123(4):305-13. doi: 10.1007/s11547-017-0828-5. PubMed PMID: 29164365.

2. Melhem E, Assi A, El Rachkidi R, Ghanem I. EOS(®) biplanar X-ray imaging: concept, developments, benefits, and limitations. J Child Orthop. 2016;10(1):1-14. doi: 10.1007/s11832-016-07130. PubMed PMID: 26883033. PubMed PMCID: PMC4763151.

3. Hui SCN, Chu WCW. Supplementary Addendum to "Radiation dose of digital radiography (DR) versus micro-dose $x$-ray (EOS) on patients with adolescent idiopathic scoliosis: 2016 SOSORTIRSSD "John Sevastic Award" Winner in Imaging Research". Scoliosis Spinal Disord. 2018;13:1. doi: 10.1186/s13013-017-0148-5. PubMed PMID: 29435497. PubMed PMCID: PMC5795282.

4. Law M, Ma WK, Chan E, Lau D, Mui C, Cheung K, Yip L, Lam W. Evaluation of cumulative effective dose and cancer risk from repetitive full spine imaging using EOS system: Impact to adolescent patients of different populations. Eur J Radiol. 2017;96:1-5. doi: 10.1016/j.ejrad.2017.09.006. PubMed PMID: 29103466.

5. Pedersen PH, Petersen AG, Østgaard SE, Tvede- brink T, Eiskjær SP. EOS micro-dose protocol: first full-spine radiation dose measurements in anthropomorphic phantoms and comparisons with EOS standard-dose and conventional digital radiology. Spine. 2018;43(22):E1313-21. doi: 10.1097/BRS.0000000000002696. PubMed PMID: 30383725.

6. Chiron P, Demoulin L, Wytrykowski K, Cavaignac E, Reina N, Murgier J. Radiation dose and magnification in pelvic X-ray: EOS ${ }^{\mathrm{TM}}$ imaging system versus plain radiographs. Orthop Traumatol Surg Res. 2017;103(8):1155-59. doi: 10.1016/j. otsr.2017.07.018. PubMed PMID: 28942025.

7. Ben Abdennebi A, Aubry S, Ounalli L, Fayache MS, Delabrousse E, Petegnief Y. Comparative dose levels between CT-scanner and slot-scanning device (EOS system) in pregnant women pelvimetry. Phys Med. 2017;33:77-86. doi: 10.1016/j. ejmp.2016.12.008. PubMed PMID: 27993442.

8. Yvert M, Diallo A, Bessou P, Rehel JL, Lhomme E, Chateil JF. Radiography of scoliosis: Comparative dose levels and image quality between a dynamic flat-panel detector and a slot-scanning device (EOS system). Diagn/nterv Imaging. 2015;96(11):117788. doi: 10.1016/j.diii.2015.06.018. PubMed PMID: 26282052.

9. Delin C, Silvera S, Bassinet C, Thelen P, Rehel JL, Legmann $P$, Folinais $D$. Ionizing radiation doses during lower limb torsion and anteversion measurements by EOS stereoradiography and computed tomography. Eur J Radiol. 2014;83(2):371-7. doi: 10.1016/j.ejrad.2013.10.026. PubMed PMID: 24291000.

10. Abrisham SMJ, Bouzarjomehri F, Nafisi-Moghadam R, Sobhan MR, Gadimi M, Omidvar F. A comparison of patients absorption doses with bone deformity due to the EOS imaging and digital radiology. Arch Bone Jt Surg. 2017;5(3):145-48. PubMed PMID: 28656161. PubMed PMCID: PMC5466858.

11. Tapiovaara M, Siiskonen T. PCXMC, A Monte Carlo program for calculating patient doses in medical X-ray examinations. Report STUK-A139; Helsinki, Finland: Radiation and Nuclear Safety Authority; 2008.

12. ICRP. The 2007 recommendations of the International Commission on Radiological Protection: ICRP publication 103. Ann ICRP. 2007;37(2-4):1332. doi: 10.1016/j.icrp.2007.10.003. PubMed PMID: 18082557.

13. Alrehily F, Hogg P, Twiste M, Johansen S, Tootell A. Scoliosis imaging: An analysis of radiation risk in the CT scan projection radiograph and a comparison with projection radiography and EOS. 
Radiography. 2019;25(3)68-74. doi: 10.1016/j. radi.2019.02.005. PubMed PMID: 31301794.

14. Escott BG, Ravi B, Weathermon AC, Acharya J, Gordon CL, Babyn PS, et al. EOS low-dose radiography: a reliable and accurate upright assessment of lower-limb lengths. The Journal of Bone \& Joint Surgery. 2013;95(23) e1831-7. doi: 10.2106/ JBJS.L.00989. PubMed PMID: 24306706.
15. Hui SCN, Pialasse JP, Wong JYH, Lam T, Ng BKW, Cheng JCY, et al. Radiation dose of digital radiography (DR) versus micro-dose x-ray (EOS) on patients with adolescent idiopathic scoliosis: 2016 SOSORT- IRSSD "John Sevastic Award" Winner in Imaging Research. Scoliosis Spinal Disord. 2016;11:46. doi: 10.1186/s13013-016-0106-7. PMCID: PMC5198497. PubMed PMID: 28035336. 